\title{
Correction to: Humoral Immunity in Mice Transplanted with Hematopoietic Stem Cells Derived from Human Umbilical Cord Blood Recapitulates That of Human Infants, by Walker JA, Vuyyuru R, Manser T, and Alugupalli KR. Stem Cells Dev 2017;26:1715-1723 DOI: 10.1089/scd.2017.0156
}

\footnotetext{
The authors of this article, Humoral Immunity in Mice Transplanted with Hematopoietic Stem Cells Derived from Human Umbilical Cord Blood Recapitulates That of Human Infants, wish to correct errors that appeared in the legend for Figure 2 and in Figure 5B. Both figures with their corresponding legends appear below in their corrected versions.
}

There were no significant differences seen in bacteremia "for anti-B. hermsii IgM responses, from day 11 to 15 ." Thus, this text has been removed from the Figure 2 legend.

In Figure $5 \mathrm{~B}$, the $\mathrm{X}$ axis was incorrectly listed as 1, 2, 3 and it has now been revised to 0,7 , and 14, days post-immunization.

The online version of this article has been corrected to reflect these corrections.

The authors sincerely regret these errors.
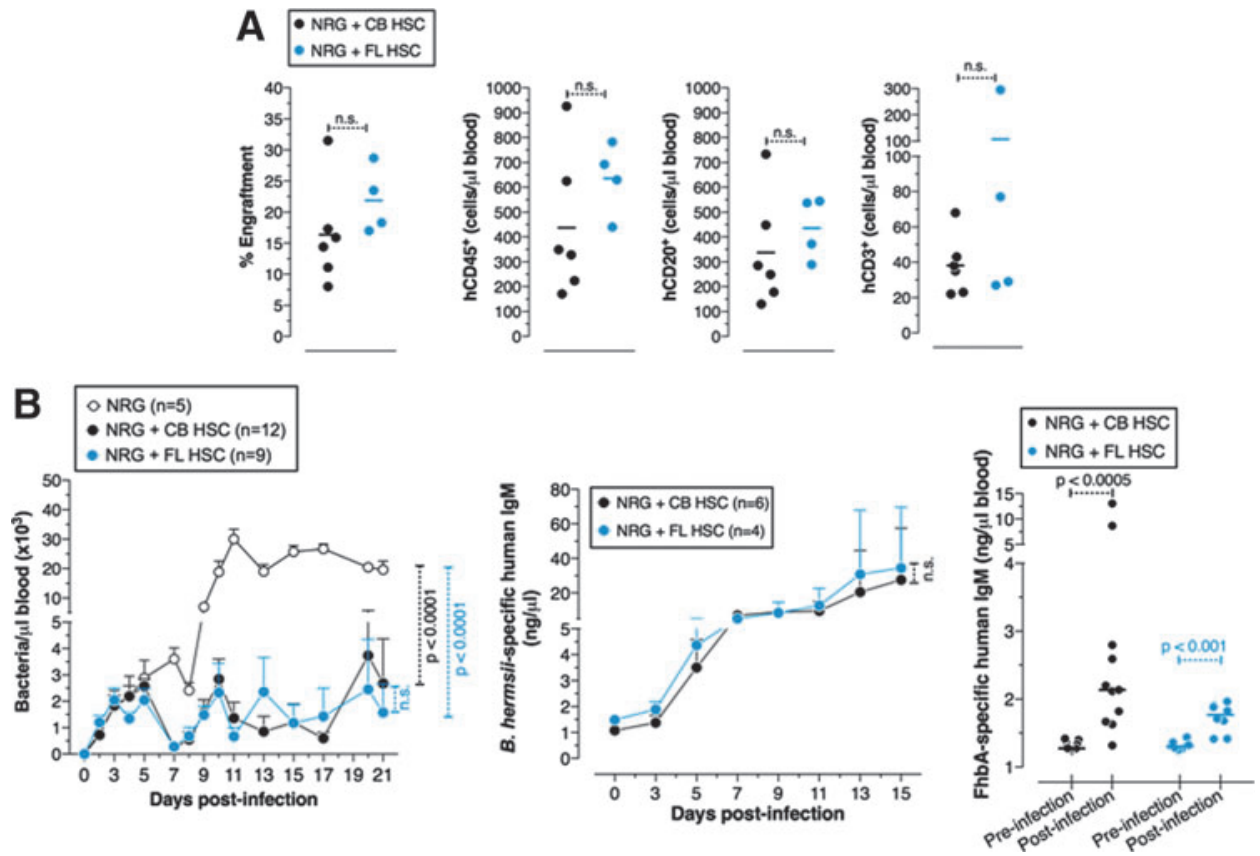

FIG. 2. Comparable reconstitution of protective immune responses in mice transplanted with human umbilical cord blood and fetal liver HSCs. (A) The relative reconstitution of human lymphocytes in the peripheral blood is referred to as percent engraftment. (B) Mice were infected with $5 \times 10^{4} B$. hermsii bacteria i.p., and bacteremia and anti-B. hermsii responses at the indicated days postinfection were determined by dark-field microscopy and ELISA, respectively. Specific IgM responses to B. hermsii outer membrane protein (FhbA) were measured on 14 days postinfection. The difference in lymphocyte reconstitution was analyzed using Student's $t$-test (two-tailed). Statistics were done using two-way ANOVA with Bonferroni posttest. Statistically significant differences in bacteremia were seen from day 10 to 21 postinfection. For bacteremia and the antibody responses the mean \pm SEM is shown. Pooled data from two independent experiments are shown. n.s., not significant. Color images available online at www.liebertpub.com/scd
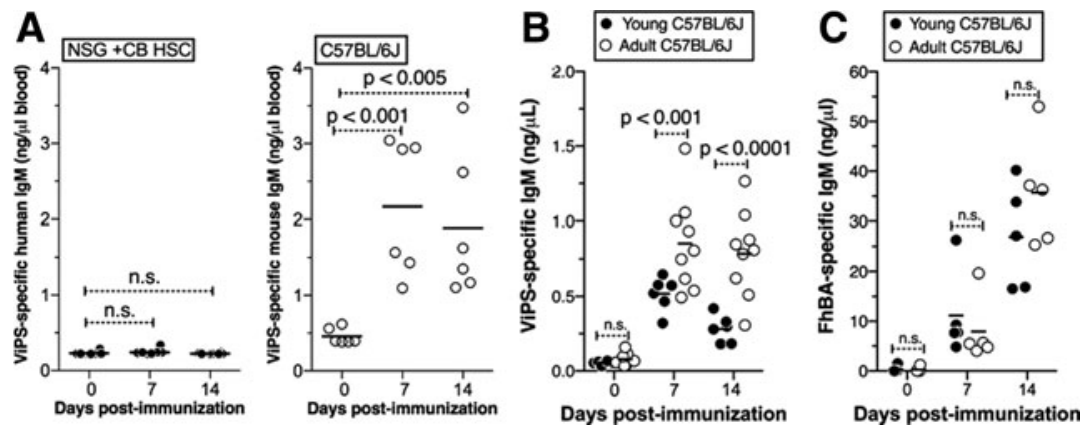

FIG. 5. Mice reconstituted with human umbilical cord blood HSCs do not respond to bacterial polysaccharide antigens. (A) Mice transplanted with human umbilical cord blood HSCs or adult C57BL6 mice were immunized with $3 \times 10^{8}$ heat-killed $S$. typhi strain Ty2 and ViPS-specific human and mouse IgM responses were measured by ELISA. Three-week-old (young) or 14-week-old (adult) mice were (B) immunized with ViPS or (C) infected with $B$. hermsii and anti-ViPS IgM and anti-FhBA responses were measured by ELISA. ViPS, Vi polysaccharide. n.s., not significant. 